\title{
Comparison of percentage excess weight loss after laparoscopic sleeve gastrectomy and laparoscopic adjustable gastric banding
}

\author{
Andrzej Lehmann ${ }^{1}$, Maciej Bobowicz ${ }^{2}$, Paweł Lech ${ }^{3}$, Michał Orłowski ${ }^{1}$, Wiaczesław Siczewski ${ }^{1}$, Maciej Pawlak ${ }^{1}$, \\ Dariusz Świetlik ${ }^{4}$, Mieczysław Witzling ${ }^{1}$, Maciej Michalik ${ }^{1,3}$ \\ ${ }^{1}$ General and Vascular Surgery Department, Ceynowa Hospital, Wejherowo, Poland \\ ${ }^{2}$ Department of Oncological Surgery, Medical University of Gdansk, Gdansk, Poland \\ ${ }^{3}$ Department of General and Minimally Invasive Surgery, University of Warmia and Mazury, Olsztyn, Poland \\ ${ }^{4}$ Faculty of Medical Informatics and Biostatistics, Medical University of Gdansk, Gdansk, Poland
}

Videosurgery Miniinv 2014; 9 (3): 351-356 DOI: $10.5114 /$ wiitm.2014.44257

\begin{abstract}
Introduction: Laparoscopic sleeve gastrectomy (LSG) and laparoscopic adjustable gastric banding (LAGB) are acceptable options for primary bariatric procedures in patients with body mass index (BMI) $35-55 \mathrm{~kg} / \mathrm{m}^{2}$.

Aim: The aim of this study is to compare the effects of these two bariatric procedures 6, 12 and 24 months after surgery. Material and methods: Two hundred and two patients were included 72 LSG and 130 LAGB patients. The average age was $38.8 \pm 11.9$ and $39.4 \pm 10.4$ years in LSG and LAGB groups, with initial BMI of $44.1 \mathrm{~kg} / \mathrm{m}^{2}$ and $45.2 \mathrm{~kg} / \mathrm{m}^{2}, p=N S$. Results: The mean percentage of excess weight loss (\%EWL) at 6 months for LSG vs. LAGB was $36.3 \%$ vs. $30.1 \%$ ( $p=$ $0.01)$ and at 12 months was $43.8 \%$ vs. 34.6\% ( $p=0.005)$. The greatest difference in the mean \%EWL at 12 months was observed in patients with initial BMI of $40-49.9 \mathrm{~kg} / \mathrm{m}^{2}$ in favor of $L S G(47.5 \% \mathrm{vs} .35 .6 \% ; p=0.01)$. Two years after surgery there was no advantage of $L S G$ and in the subgroup of patients with $B M I 50-55 \mathrm{~kg} / \mathrm{m}^{2}$ there was a trend in favor of LAGB (57.2\% vs. 30\%; $p=0.07)$. The multiple regression model of independent variables (age, gender, initial BMI and the presence of comorbidities) proved insignificant in prediction of the best outcome in means of \%EWL for either operative modality. None of these factors in the logistic regression model could determine the type of surgery that should be used in particular patients.

Conclusions: During the first 2 years after surgery, the best results were obtained in women with lower BMI undergoing LSG surgery. The LSG provides greater \%EWL after a shorter period of time though the difference decreases in time.
\end{abstract}

Key words: obesity, laparoscopic sleeve gastrectomy, excess weight loss, comparison, laparoscopic adjustable gastric banding, treatment outcomes.

\section{Introduction}

Surgical treatment of obesity reduces excess body weight by $50-70 \%$ when compared to behavioral and pharmacological therapies [1]. Sleeve gastrectomy (SG) and adjustable gastric banding (AGB) are the most popular restrictive methods used in the surgical treatment of obesity [2, 3].
Sleeve gastrectomy is the first step of a duodenal switch and was first described by Hess in 1988 [4]. Thirteen years ago, Ren et al. performed the first laparoscopic gastric sleeve resection, also as a first stage of a biliopancreatic diversion with duodenal switch (BPD-DS), and it became an independent procedure treatment soon after [5]. 
The idea of adjustable gastric banding appeared in the late 1980s [6-8]. Laparoscopic AGB (LAGB) is a restrictive procedure and more importantly it is one of the few reversible bariatric procedures and was first described in 1994 by Belachew et al. [9]. It is one of the most popular bariatric procedures in Europe and Australia and in recent years it has slowly been gaining popularity in the USA. The pioneers of laparoscopic AGB implantation included Guy-Bernard Cadiere and Franco Favretti. In Poland, this method was used for the first time in 1998 [10].

Both LSG and LAGB have their pros and cons. Both have well-documented advantages and disadvantages but there are few reports directly comparing the outcomes of these two operative modalities. Therefore, we decided to undertake a study evaluating the outcomes of LAGB and LSG in terms of \% of excess weight loss (\%EWL) 6, 12, and 24 months after surgery. As there are no clear guidelines suggesting which method is better for which patients, we applied a univariate and multivariate regression model to factors that might become part of a predictive model of outcome in patients with body mass index (BMI) in the range of $35-55 \mathrm{~kg} / \mathrm{m}^{2}$.

\section{Material and methods}

\section{Patients}

Data on basic characteristics (age, gender, initial weight, BMI and comorbidities) (Table I), and \%EWL at 6,12 and 24 months after surgery were extracted from 2 previously published studies for the population of patients with morbid obesity with BMI $>40 \mathrm{~kg} / \mathrm{m}^{2}$ or $\mathrm{BMI}>35 \mathrm{~kg} / \mathrm{m}^{2}$ with significant comorbidities, and up to $\mathrm{BMI}=55 \mathrm{~kg} / \mathrm{m}^{2}$, who underwent operations between the years 2005 and 2009 in our institution $[11,12]$. Two hundred and two consecutive patients who underwent LSG (72 patients) or LAGB (130 patients) fulfilled the above criteria and were included in this study. The type of surgery to be undertaken was chosen jointly by the bariatric team and the patient after the multistep qualification. The operative techniques were described elsewhere [11-13]. The flow chart of participation in follow-up visits is shown in Figure 1.

\section{Statistical analysis}

Statistical calculations were performed using Statistica 9.1 computer software from StatSoft, Inc. Krakow, Poland, by an independent statistician. Chisquare $\left(\chi^{2}\right)$ test, Student'st-test, Shapiro-Wilk distribution analysis, Mann-Whitney U-test, post hoc ANOVA analysis and the Kruskal-Wallis ANOVA test and Dunn's multiple comparisons were used. Statistical significance was established at the $p<0.05$ level.

\section{Results}

The mean age of patients in the LSG group was $38.8 \pm 11.9$ years (range: 19-67) and in the LAGB group was $39.4 \pm 10.4$ (range: $22-74$ ). The mean BMI was $44.1 \pm 5.3$ (range: $35.1-54.7$ ) and $45.2 \pm 5.4$ (range: $35.2-54.8)$ respectively $(p=0.17)$. Gender distribution was similar in both groups and around $58 \%$ of patients in each group had at least one comorbid condition, as shown in Table I.

The mean \%EWL at 6 and 12 months was statistically more significant in the LSG group. The \%EWL equaled 36.3 at 6 months and 43.8 at 12 months whereas in the LAGB group it was 30.1 and 34.6 respectively. The average \%EWL at 24 months in patients who underwent an LSG operation was 44.0

Table I. Patients' characteristics

\begin{tabular}{|lccc|}
\hline Parameter & LSG & LAGB & Value of $p$ \\
\hline Number of patients & 72 & 130 & NS \\
\cline { 1 - 3 } Female, $n(\%)$ & $55(76.4)$ & $99(76.1)$ & \\
\hline Male, $n(\%)$ & $17(23.6)$ & $31(23.9)$ & NS \\
\hline Age, mean \pm SD (range) [years] & $38.8 \pm 11.9(19-67)$ & $39.4 \pm 10.4(22-74)$ & NS \\
\hline Initial weight, mean \pm SD (range) $[\mathrm{kg}]$ & $126.7 \pm 20.6(90-170)$ & $129.7 \pm 22.3(90-207)$ & NS \\
\hline Initial BMI, mean \pm SD (range) $\left[\mathrm{kg} / \mathrm{m}^{2}\right]$ & $44.1 \pm 5.3(35.1-54.7)$ & $45.2 \pm 5.4(35.2-54.8)$ & NS \\
\hline Patients with comorbidities, $n(\%)$ & $42(58.3)$ & $76(58.5)$ & \\
\hline
\end{tabular}

NS - not significant 


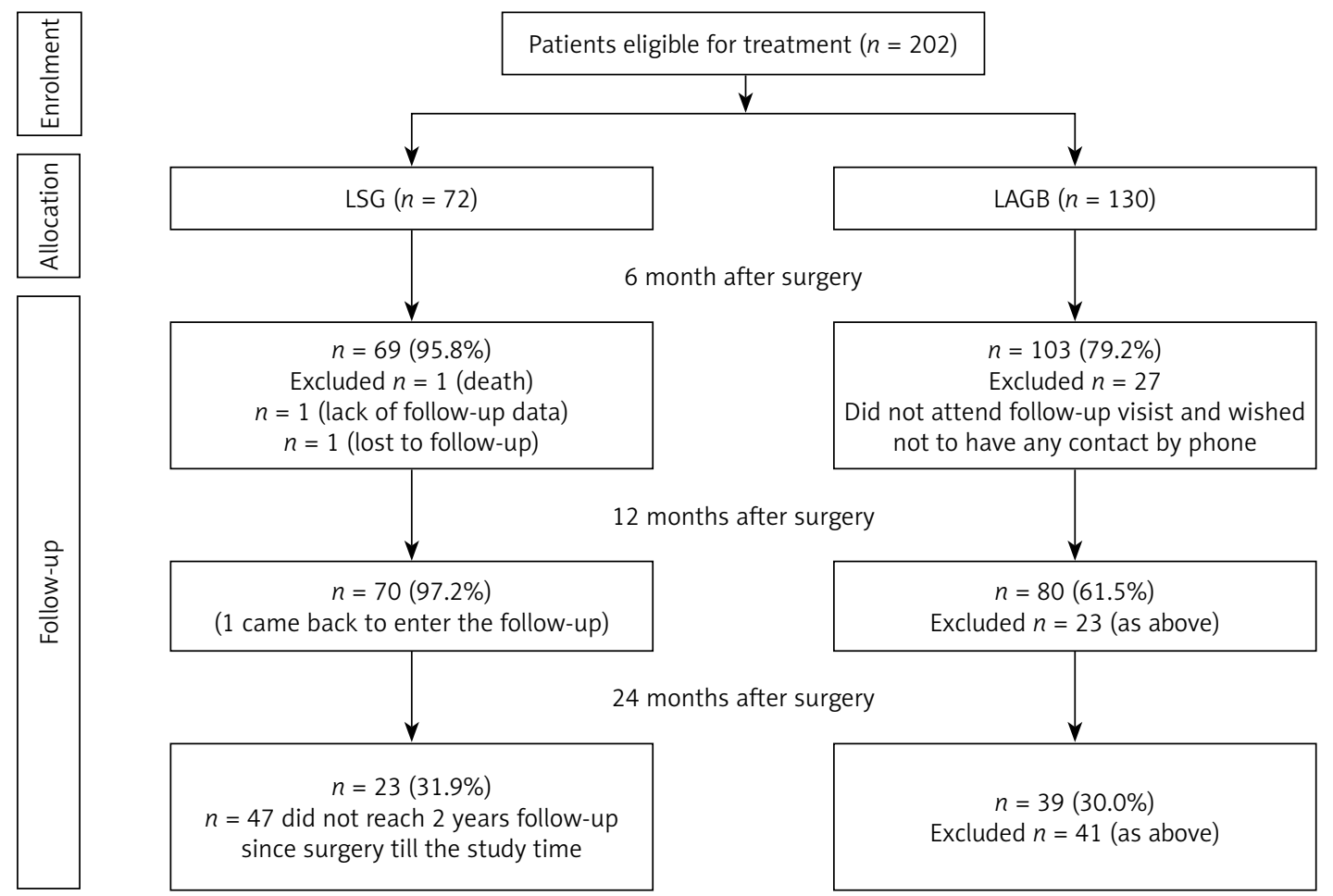

Figure 1. Participation in follow-up visits

and was 37.2 in LAGB patients, though the difference was not significant. Table II shows the effect of the type of operation on the \%EWL at the particular times of observation.

We stratified the patients into three subgroups: grade II obesity (BMI 35-39.9 kg/m²), morbid obesity (BMI 40-49.9 kg/m²) and super-obesity (BMI 50-55 kg/ $\mathrm{m}^{2}$ ) to see which patients benefited the most from both types of surgery. The results are presented in Table III.

On the other hand, both operative techniques had similar complication rates when assessed according to the BAROS scoring system, with significantly more minor medical complications following the LSG procedure [14] (Table IV).

Age, gender, initial BMI and the presence of comorbidities proved insignificant in the multiple re-
Table II. \%EWL 6, 12 and 24 months after surgery

\begin{tabular}{|lccc|}
\hline Time [months] & LSG & LAGB & Value of $p$ \\
\hline 6 & $36.3 \pm 15.5$ & $30.1 \pm 16.6$ & 0.01 \\
\hline 12 & $43.8 \pm 18.9$ & $34.6 \pm 20.8$ & 0.005 \\
\hline 24 & $44.0 \pm 23.8$ & $37.2 \pm 24.0$ & 0.28 \\
\hline
\end{tabular}

gression model of independent variables that might have had influence on the postoperative loss of the excess weight. Neither age, gender, initial BMI nor the presence of comorbidities was an independent factor in the logistic regression model that could determine the type of surgery that should be used in particular patients.

Table III. Average \%EWL for initial BMI subgroups at 6, 12 and 24 months after surgery

\begin{tabular}{|lccccccccc|}
\hline BMI [kg/m $\left.{ }^{2}\right]$ & \multicolumn{3}{c}{$35-39.9$} & & $40-49.9$ & & $50-55$ \\
\hline Time [months] & 6 & 12 & 24 & 6 & 12 & 24 & 6 & 12 & 33.9 \\
\hline LSG & 36.0 & 42.5 & 51.0 & 39.1 & 47.5 & 48.4 & 28.4 & 30.0 \\
\hline LAGB & 32.9 & 33.7 & 23.1 & 31.1 & 35.6 & 35.4 & 24.9 & 32.8 & 57.2 \\
\hline Value of $p$ & NS & NS & 0.07 & 0.03 & 0.01 & NS & NS & NS \\
\hline
\end{tabular}


Table IV. Complication rates after LSG and LAGB procedures

\begin{tabular}{|lccc|}
\hline Complications & LSG & LAGB & Value of $p$ \\
\hline Major surgical & $4(5.6 \%)$ & $12(9.2 \%)$ & NS \\
\hline Minor surgical & $5(6.9 \%)$ & $10(7.7 \%)$ & NS \\
\hline Major medical & $3(4.2 \%)$ & $3(2.3 \%)$ & NS \\
\hline Minor medical & $12(16.7 \%)$ & $0(0.0 \%)$ & 0.0001 \\
\hline Conversions & $0(0.0 \%)$ & $2(1.5 \%)$ & NS \\
\hline
\end{tabular}

\section{Discussion}

Laparoscopic sleeve gastrectomy and gastric banding are the two most frequently performed bariatric procedures in Europe. They are well-developed and standardized procedures. The main differences are the reversibility, the need for adjustments, the type of complications and the magnitude of their outcomes. The main advantage of LAGB surgery is that it is reversible and the band can be removed at any time. It can also be adjusted by injecting more fluid into the port to tighten it, thus providing more restriction if needed. The LSG procedure provides good restriction, and by eliminating the greater curvature and therefore the majority of ghrelin-producing cells, it has an influence on the hunger-satiety axis, though one that is irreversible.

Short-term outcomes of both procedures were previously documented by other authors who deemed a loss of more than $50 \%$ of excess weight a success and loss of less than $25 \%$ a failure [15-19]. Several other factors were considered to be also important in assessing the outcomes of bariatric procedures, such as the number and severity of complications, resolution of comorbidities, improvement in self-esteem, social life, daily activities, and sex life [14]. Although very objective, the proposed scales are frequently ignored by patients who do not want to expose their private life. This is why we decided to compare LSG and LAGB with regards to only the $\% E W L$ and the proportion of complications.

In general we can say that LSG was more beneficial in the short term perspective of 1 year after surgery. Patients who underwent this procedure had significantly greater \% excess weight loss at 6 and 12 months after surgery than the patients in the LAGB group. Even though this effect was still present at 24 months, the difference was not statistical- ly significant. We can say that based on our results, laparoscopic sleeve gastrectomy is more beneficial than laparoscopic adjustable gastric banding in the perspective of 2 years after the surgery under the condition that the patient is willing to accept the irreversibility of the procedure.

When we assessed the impact of LSG and LAGB on the \%EWL with regards to the BMI subgroups, we could observe a greater \%EWL after LSG at 6, 12 and 24 months in patients with a BMI in the range of $35-39.9 \mathrm{~kg} / \mathrm{m}^{2}$ and $40-49.9 \mathrm{~kg} / \mathrm{m}^{2}$. However, a statistically significant difference in \%EWL was observed only in patients with a BMI of $40-49.9 \mathrm{~kg} /$ $\mathrm{m}^{2}$ at 6 and 12 months. Interestingly, patients with super-morbid obesity had similar \%EWL 6 and 12 months after LSG and LAGB surgery. Even more interestingly, it seems that in the long perspective super-obese patients might benefit more from LAGB than LSG. Our patients with super-morbid obesity had almost a two-fold greater \%EWL at 24 months after LAGB than the LSG surgery, and probably the difference would reach statistical significance if the analyzed groups were bigger. We think that this twofold difference in \%EWL at 24 months after surgery might derive from the fact that the adjustable gastric band is adjusted every 3 months to maintain the therapeutic effect and to facilitate further weight loss, and in this respect it might be more beneficial in this group of patients. On the other hand, the LSG procedure provides a more rapid excess weight loss, mainly in the initial 12 months, with a gradual extension of the gastric sleeve afterwards and the possibility to facilitate greater amounts of food, thus decreasing the potential to eliminate the excess weight loss. At the same time it deregulates the hormonal axis responsible for the hunger-satiety sensation, causing a decrease in hunger and thus reducing the amounts of ingested food. This was reflected in patients with a BMI of $35-39.9 \mathrm{~kg} / \mathrm{m}^{2}$ with comorbidities, who present an opposite trend to super-obese patients, with LSG providing a more than two-fold greater reduction of excess body weight at 24 months after surgery.

Even though it was a retrospective analysis performed on relatively small groups, the populations were almost homogeneous in terms of initial BMI, age and gender distribution, like in case-matched studies. All patients were operated on by a small bariatric team. Therefore, these differences can be attributed to the type of operation and not selection bias. 
Having the above results we were interested to know if any of the preoperative factors such as age, gender, BMI, or the presence of comorbidities might determine the outcome of the procedure. A logistic regression model showed that none of the above factors have any prognostic value for the LSG and LAGB procedure outcomes. We also checked via a multivariate regression analysis if any of the above factors might influence the choice of the most appropriate operation for particular patients. Apparently neither age, gender, BMI nor the presence of comorbidities determines which procedure to choose.

We still have to choose the type of surgery based on our intuition and the patient's preference. We can base our judgment on EAES and SAGES guidelines but there is no definite indication for any type of surgery, especially in the era of rapidly appearing new procedures such as mini-gastric bypass, the endoscopic bypass systems or new technologies like Tantalus [20-22]. Probably further multi-center studies on greater numbers of patients might elicit predictive factors for the outcomes of bariatric surgery. With time we might also learn about the factors that will make the choice between different metabolic procedures easier, and based on calculations be able to predict which procedure might be the most beneficial for our patient, leading to a more patient-tailored strategy in metabolic disorders.

\section{Conclusions}

Both methods show an acceptable percentage of excess weight loss with acceptable complication rates. During the first 2 years after surgery, the best results were obtained in women with lower BMI undergoing LSG surgery. Laparoscopic sleeve gastrectomy provides greater \%EWL after a shorter period of time, though the difference decreases in time. In patients with $\mathrm{BMI}>50 \mathrm{~kg} / \mathrm{m}^{2}$, LAGB might be a better option for longitudinal control of obesity. However, in order to prove the superiority of one method over another, further and more detailed studies need to be conducted.

\section{Acknowledgments}

The first two authors contributed equally to this work.

The authors would like to thank Marta Kedrzycki, English Division, Medical University of Gdansk, for her great help with the manuscript preparation and language corrections.

\section{References}

1. Elder KA, Wolfe BM. Bariatric surgery: a review of procedures and outcomes. Gastroenterology 2007; 132: 2253-71.

2. Paluszkiewicz R, Kalinowski P, Wróblewski T, et al. Prospective randomized clinical trial of laparoscopic sleeve gastrectomy versus open Roux-en-Y gastric bypass for the management of patients with morbid obesity. Videosurgery Miniinv 2012; 7: 225-32.

3. Buchwald H, Oien DM. Metabolic/bariatric surgery worldwide 2008. Obes Surg 2009; 19: 1605-11.

4. Hess DS, Hess DW. Biliopancreatic diversion with a duodenal switch. Obes Surg 1998; 8: 267-82.

5. Ren CJ, Patterson E, Gagner M. Early results of laparoscopic biliopancreatic diversion with duodenal switch: a case series of 40 consecutive patients. Obes Surg 2000; 10: 514-23.

6. Kuzmak LI. A review of seven years' experience with silicone gastric banding. Obes Surg 1991; 1: 403-8.

7. Szinicz G, Müller L, Erhart W, et al. "Reversible gastric banding" in surgical treatment of morbid obesity - results of animal experiments. Res Exp Med (Berl.) 1989; 189: 55-60.

8. Forsell P, Hallberg D, Hellers G. A gastric band with adjustable inner diameter for obesity surgery: preliminary studies. Obes Surg 1993; 3: 303-6.

9. Belachew M, Legrand MJ, Defechereux TH, et al. Laparoscopic adjustable gastric banding in the treatment of morbid obesity: a preliminary report. Surg Endosc 1994; 8: 1354-6.

10. Wylezoł M, Pardela M, Gluck M, et al. Swedish adjustable gastric band (SAGB) implanted laparoscopically in the treatment of morbid obesity - the first experience in Poland. Med Sci Monit 2000; 6: 441-5.

11. Bobowicz M, Lehmann A, Orlowski M, et al. Preliminary outcomes 1 year after laparoscopic sleeve gastrectomy based on Bariatric Analysis and Reporting Outcome System (BAROS). Obes Surg 2011; 21: 1843-8.

12. Michalik M, Lech P, Bobowicz M, et al. A 5-year experience with laparoscopic adjustable gastric banding: focus on outcomes, complications, and their management. Obes Surg 2011; 21: 1682-6.

13. Szydłowski K, Michalik M, Pawlak M, Bobowicz M, Frask A. Band misplacement: a rare complication of laparoscopic adjustable gastric banding. Videosurgery Miniinv 2012; 7: 40-4.

14. Oria HE, Moorehead MK. Updated Bariatric Analysis and Reporting Outcome System (BAROS). Surg Obes Relat Dis 2009; 5: 60-6.

15. Steffen R, Potoczna N, Bieri N, Horber FF. Successful multiintervention treatment of severe obesity: a 7-year prospective study with 96\% follow-up. Obes Surg 2009; 19: 3-12.

16. Holéczy P, Novák P, Králová A. 30\% complications with adjustable gastric banding: what did we do wrong? Obes Surg 2001; 11: 748-51.

17. Forsell P, Hellers G. The Swedish Adjustable Gastric Banding (SAGB) for morbid obesity: 9-year experience and 4-year follow up of patients operated with a new adjustable band. Obes Surg 1997; 7: 345-51. 
18. Bužga M, Holéczy P, Svagera Z, et al. Effects of sleeve gastrectomy on parameters of lipid and glucose metabolism in obese women -6 months after operation. Videosurgery Miniinv 2013; 8: $22-8$.

19. Mittermair RP, Aigner F, Nehoda H. Results and complications after laparoscopic adjustable gastric banding in super-obese patients, using the Swedish band. Obes Surg 2004; 14: 1327-30.

20. Sanmiguel CP, Conklin JL, Cunneen SA, et al. Gastric electrical stimulation with the TANTALUS System in obese type 2 diabetes patients: effect on weight and glycemic control. J Diabetes Sci Technol 2009; 3: 964-70.

21. Rodriguez-Grunert L, GalvaoNeto MP, Alamo M, et al. First human experience with endoscopically delivered and retrieved duodenal-jejunal bypass sleeve. Surg Obes Relat Dis 2008; 4: 55-9.

22. Tarnowski W, Stanowski E, Paśnik K, et al. Electric stimulation of the stomach in the treatment of diabetes mellitus in obese patients [Polish]. Postępy Nauk Medycznych 2009; 22 (Suppl 1).

Received: 20.10.2013, accepted: 23.01.2014 